\title{
SECTION SPACES OF REAL ANALYTIC VECTOR BUNDLES AND A THEOREM OF GROTHENDIECK AND POLY
}

\author{
DIETMAR VOGT \\ FB Math.-Nat., Bergische Universität Wuppertal \\ Gauss-Str. 20, D-42097 Wuppertal, Germany \\ E-mail:dvogt@math.uni-wuppertal.de
}

\begin{abstract}
The structure of the section space of a real analytic vector bundle on a real analytic manifold $X$ is studied. This is used to improve a result of Grothendieck and Poly on the zero spaces of elliptic operators and to extend a result of Domański and the author on the nonexistence of bases to the present case.
\end{abstract}

It was shown first by Grothendieck in the scalar case and then by Poly in the general case of a linear differential operator on a real analytic manifold acting between real analytic vector bundles, that the space of zero solutions of an elliptic operator (in the sense that the $C^{\infty}$-solutions are even real analytic) is complemented in $C^{\infty}$, resp. in the space of $C^{\infty}$-sections of a given bundle, if and only if, it is finite dimensional. In the case of a differential polynomial in dimension $\geq 2$ this implies that it is never complemented. We improve this result in the way that even complementedness in the space of real analytic sections, or in any superspace of these sections, implies finite dimensionality. For the proof we show that any complemented Fréchet subspace of a real analytic section space is finite dimensional. This generalizes a result of P. Domański and the author in 2 from the case of spaces of real analytic functions on open subsets of $\mathbb{R}^{d}$ to the present case. The results of [2] then imply that for non-compact $X$ none of the section spaces of real analytic vector bundles has a (Schauder) basis.

We use common terminology of functional analysis, see e.g. 7. and for homological concepts [12. For concepts of sheaf theory and complex function theory in several variables we refer to [4, for real analytic functions to [6].

2000 Mathematics Subject Classification: Primary 46E10; Secondary 32C05, 46A63, 46M20. Key words and phrases: real analytic manifold, differential operator, Schauder basis. The paper is in final form and no version of it will be published elsewhere. 
Let $X$ be a $d$-dimensional, connected and $\sigma$-compact real analytic manifold. By a theorem of Grauert ([3, Theorem 3]) we may assume that $X$ is a closed submanifold of $\mathbb{R}^{N}$ where $N$ is sufficiently large. Let $(E, \pi, X)$ be a $p$-dimensional real analytic vector bundle with base $X$ and $A_{E}(X)$ the space of real analytic sections on $X$.

We want to prove that every complemented Fréchet subspace of $A_{E}(X)$ is finite dimensional. In the case of a trivial bundle this can be done by a simple reduction to the results of [2]. In this case we have $A_{E}(X)=A\left(X, \mathbb{C}^{p}\right)$ where $A\left(X, \mathbb{C}^{p}\right)$ denotes the space of $\mathbb{C}^{p}$-valued real analytic functions.

Lemma 1. If $F \subset A\left(X, \mathbb{C}^{p}\right)$ is a complemented subspace, which is a Fréchet space in the induced topology, then $F$ is finite dimensional.

Proof. We assume that $X$ is a closed submanifold of $\mathbb{R}^{N}$. Then there is an open neighborhood $\Omega$ of $X$ in $\mathbb{R}^{N}$ and a real analytic retraction $\varphi$ from $\Omega$ onto $X$ (see 6. Theorem 2.7.10]). The map $f \mapsto f \circ \varphi^{-1}$ imbeds $A\left(X, \mathbb{C}^{p}\right)$ into $A\left(\Omega, \mathbb{C}^{p}\right)$ as a complemented subspace. Hence $E$ is isomorphic to a complemented Fréchet subspace of $A\left(\Omega, \mathbb{C}^{p}\right)$ and therefore finite dimensional (see [2, Theorem 3.7]). Notice that the arguments leading to 2. Theorem 3.7] also apply to $A\left(\Omega, \mathbb{C}^{p}\right)$.

The general case needs a more careful analysis.

Let $X \subset \mathbb{R}^{N}$ be a closed connected real analytic submanifold. Then there exists an open holomorphically convex subset $\Omega \subset \mathbb{C}^{N}$ and a connected complex submanifold $Y \subset \Omega$, such that $X=Y \cap \mathbb{R}^{N} . Y$ is called a complexification of $X$. We assume that we are given a coherent ${ }_{Y} \mathscr{O}$-sheaf $\mathscr{G}$ on $Y$.

Now we will describe the topology of the section space $G(X):=\Gamma(X, \mathscr{G})$. To do this in a proper way, we consider $\mathscr{G}$, by trivial extension, as a coherent sheaf on $\Omega$.

Let $I \subset X$ be compact and $U \subset \subset \Omega$ an open neighborhood of $I$. We choose a finite set of generators $s_{1}, \ldots, s_{m} \in G(\Omega)$ of $G(I)$ over $H(I)$ (see [4, Chap. VII, Sec. A, Theorem 7] together with [5, Lemma 5.4.1]). For $s \in G(I)$ we set

$$
\|s\|_{U, I}=\inf \left\{\max _{j=1, \ldots, m}\left\|f_{j}\right\|_{U}: f_{1}, \ldots, f_{m} \in H^{\infty}(U), \sum_{j=1}^{m} f_{j} s_{j}=s\right\}
$$

where the inf is understood as $+\infty$ if there is no such representation. We set

$$
G(U, I)=\left\{s \in G(I):\|s\|_{U, I}<+\infty\right\}
$$

and this defines a Banach space. We have $G(I)=\bigcup_{U} G(U, I)$, where $U$ runs through the relatively compact neighborhoods of $I$. We equip $G(I)$ with the inductive topology of the $G(U, I)$. Then it is an (LB)-space and its topology does not depend on the set of generators which we have chosen. We choose for every $I$ a fixed set of generators.

The topology on $G(X)$ is now defined as the projective topology of the $G(I)$. For any covering $I_{1} \subset \stackrel{\circ}{I}_{2} \subset I_{2} \subset \stackrel{\circ}{I}_{3} \subset \ldots$ of $X$ we have $G(X)=\operatorname{limproj} G\left(I_{n}\right)$. Therefore $G(X)$ is a (PLS)-space, that is a projective limit of (LB)-spaces with compact linking maps in the (LB)-steps, and $\operatorname{Proj}^{1} G(X)=\operatorname{Proj}^{1} G\left(I_{n}\right)$ is well defined and independent of the covering. 
Lemma 2. $\operatorname{Proj}^{1} G(X)=0$ and therefore $G(X)$ is ultrabornological.

Proof. Let $\mathcal{U}: U_{1} \subset \subset U_{2} \subset \subset \ldots$ be a covering of $X$ by connected open sets then, by the real analytic version of Cartan's Theorem B (see [1, Théorème 3]) we have $H^{1}\left(X,\left.\mathscr{G}\right|_{X}\right)=0$ and $H^{q}\left(U_{n},\left.\mathscr{G}\right|_{X}\right)=0$ for all $q \geq 1$ and $n$. Therefore, by Leray's theorem, $H^{1}\left(\mathcal{U},\left.\mathscr{G}\right|_{X}\right)=0$. Since the projective spectrum $G\left(U_{n}\right)$ is cofinal to the projective spectrum $G\left(I_{n}\right)$, this implies $\operatorname{Proj}^{1} G(X)=0$. By [11, Lemma 3.2] this implies that $G(X)$ is ultrabornological.

First we use [2, Lemma 3.3.] to prove a more general version of the same lemma.

LemmA 3. Let $I \subset J$ be connected compact sets in $X$ and $I \subset U \subset \subset \Omega, U$ open. Let $0<\alpha<1$. Then there are open sets $I \subset V \subset \subset \Omega, J \subset W \subset \subset \Omega$ and a constant $C>0$ such that for every $s \in G(X)$ with $\|s\|_{U, I}<1$ and every $r>0$ there exist $\sigma \in G(V, I)$, $\tau \in G(W, J)$ so that $s=\sigma+\tau$ on $I$ and

$$
\|\sigma\|_{V, I} \leq C \frac{1}{r^{\alpha}}, \quad\|\tau\|_{W, J} \leq C r^{1-\alpha}
$$

Proof. Let $s_{1}, \ldots, s_{m} \in G(\Omega)$ be the generators for $G(I)$ and $s \in G(X),\|s\|_{U, I}<1$. Then there are functions $f_{1}, \ldots, f_{m} \in H^{\infty}(U)$ such that $s=\sum_{j=1}^{m} f_{j} s_{j}$ and $\left\|f_{j}\right\|_{U}<1$ for all $j$.

By [2, Lemma 3.3] we find open sets $I \subset V \subset \subset \Omega, J \subset W \subset \subset \Omega$ and $C>0$ such that for every $f \in H(U)$ with $\|f\|_{U} \leq 1$ and every $r>0$ there exist $g \in H(V), h \in H(W)$ so that $f=g+h$ on $I$ and

$$
\|g\|_{V} \leq C \frac{1}{r^{\alpha}}, \quad\|h\|_{W} \leq C r^{1-\alpha} .
$$

We apply this to $f_{1}, \ldots, f_{m}$ and obtain $g_{1}, \ldots, g_{m} \in H^{\infty}(V)$ and $h_{1}, \ldots, h_{m} \in$ $H^{\infty}(W)$. We set

$$
\sigma=\sum_{j=1}^{m} g_{j} s_{j}, \quad \tau=\sum_{j=1}^{m} h_{j} s_{j}
$$

It follows immediately that $\|\sigma\|_{V, I} \leq C \frac{1}{r^{\alpha}}$.

If $\sigma_{1}, \ldots, \sigma_{M}$ are the generators for $G(J)$, then we have expansions $s_{j}=\sum_{k=1}^{M} a_{j, k} \sigma_{k}$ with $a_{j, k} \in H^{\infty}\left(W_{0}\right)$, where $J \subset W_{0} \subset \subset \Omega$ is open. We may assume that $W \subset W_{0}$, otherwise we replace $W$ by $W \cap W_{0}$. Inserting these expansions into the formula for $\tau$ we obtain by straightforward estimates $\|\tau\|_{W, J} \leq C^{\prime} r^{1-\alpha}$ with a new constant $C^{\prime}>0$.

We proceed now as in [2] after Lemma 3.3.

A Fréchet space with a fundamental sequence of seminorms $\left(\|\cdot\|_{n}\right)$ defining the topology is said to have property $(\overline{\bar{\Omega}})$ if

$$
\forall k \exists m \forall n, \vartheta \in] 0,1\left[\exists C \forall u \in E^{\prime} \quad\|u\|_{m}^{*} \leq C\|u\|_{k}^{* \vartheta}\|u\|_{n}^{* 1-\vartheta},\right.
$$

Here $\|\cdot\|^{*}$ denotes the dual norm for $\|\cdot\|$.

REMARK. A Fréchet space $E$ has $(\overline{\bar{\Omega}})$ if and only if

$$
\forall U_{1} \exists U_{2} \forall U_{3}, \gamma>0 \exists C \forall r>0 \quad U_{2} \subset C\left(\frac{1}{r^{\gamma}} U_{1}+r U_{3}\right),
$$

where $U_{j}, j=1,2,3$ are 0 -neighbourhoods in $E$. 
Arguing, on the basis of Lemma 3, precisely as in the proof of [2, Theorem 3.4] we obtain:

ThEOREM 4. Every Fréchet space $E$ which is a quotient of $G(X)$ has property $(\overline{\bar{\Omega}})$.

To investigate the topological properties of Fréchet spaces imbedded into $G(X)$ we have to strengthen our assumptions. We assume that $\mathscr{G}$ is locally free (see 4, Chap. VII, Sec. C]).

We need the following linear topological concept. A Fréchet space with a fundamental sequence of seminorms $\left(\|\cdot\|_{n}\right)$ defining the topology is said to have property $(\underline{D N})$ if

$$
\exists n \forall k \exists l, C>0, \tau \in] 0,1\left[\quad\|x\|_{k} \leq C\|x\|_{n}^{\tau}\|x\|_{l}^{1-\tau}\right.
$$

for every $x \in E$.

Lemma 5. If $Y$ is a connected Stein manifold and $\mathscr{G}$ a locally free sheaf, then $G(Y)$ has property $(\underline{D N})$.

Proof. Let $Y_{1}, Y_{2}, \ldots$ be a covering of $Y$ by relatively compact open sets such that $\left.\mathscr{G}\right|_{Y_{j}} \cong$ $\left.\mathscr{O}^{p}\right|_{Y_{j}}$. Therefore $G\left(Y_{j}\right) \cong H\left(Y_{j}, \mathbb{C}^{p}\right)$. Let $\varphi_{j}: G\left(Y_{j}\right) \rightarrow H\left(Y_{j}, \mathbb{C}^{p}\right)$ be the isomorphism. By possibly shrinking the $Y_{j}$ we may assume that $\varphi_{j}(G(Y)) \subset H^{\infty}\left(Y_{j}, \mathbb{C}^{p}\right)$. We may also assume that all $Y_{j}$ are hyperconvex, e.g. we can choose them as polydiscs under some local chart. An open bounded set $U$ is called hyperconvex whenever it is connected and there is a continuous plurisubharmonic negative function $\rho$ on $U$ such that the sets $\{z \in U: \rho(z)<c\}$ are relatively compact in $U$ for every negative $c$, see [5, p. 80].

We choose a sequence $V_{1}, V_{2}, \ldots$ of open sets such that $V_{j} \subset \subset Y_{j}$ for all $j$ and still $\bigcup_{j} V_{j}=Y$. Since $Y$ is connected we may assume that the $V_{j}$ are ordered in a way that $\left(\bigcup_{j=1}^{n} V_{j}\right) \cap V_{n+1} \neq \emptyset$ for all $n$.

For $f=\left(f_{1}, \ldots, f_{p}\right) \in H\left(Y_{j}, \mathbb{C}^{p}\right)$ we put $\|f\|_{V_{j}}=\sup \left\{\left|f_{j}(z)\right|: z \in V_{j}, j=1, \ldots, p\right\}$, and for $s \in G(Y)$

$$
\|s\|_{n}=\sup _{j=1, \ldots, n}\left\|\varphi_{j}(s)\right\|_{V_{j}} .
$$

The norms $\|\cdot\|_{n}$ are a fundamental system of seminorms for the Fréchet space $G(Y)$. In particular for any $n$ there is $m_{n} \geq n$ and $C_{n} \geq 1$ such that

$$
\sup _{j=1, \ldots, n}\left\|\varphi_{j}(s)\right\|_{Y_{j}} \leq C_{n}\|s\|_{m_{n}} .
$$

We show that there is a constant $C_{n}^{\prime} \geq C_{n}$ and $0<\tau_{n}<1$ such that

$$
\|s\|_{n} \leq C_{n}^{\prime}\|s\|_{n-1}^{\tau_{n}}\|s\|_{m_{n}}^{1-\tau_{n}}
$$

for all $s \in G(Y)$.

If $\|s\|_{n}=\left\|\varphi_{j}(s)\right\|_{V_{j}}$ where $j \leq n-1$, this inequality is trivial. So we assume that $\|s\|_{n}=\left\|\varphi_{n}(s)\right\|_{V_{n}}$. We set $W_{n}=\left(\bigcup_{j=1}^{n-1} V_{j}\right) \cap V_{n}$. Then from [13, Proposition 1.4.2] we conclude that there is $0<\tau_{n}<1$ such that

$$
\|f\|_{V_{n}} \leq\|f\|_{W_{n}}^{\tau_{n}}\|f\|_{Y_{n}}^{1-\tau_{n}}
$$

for all $f \in H\left(V_{n}, \mathbb{C}^{p}\right)$. This implies

$$
\|s\|_{n} \leq C_{n}\left\|\varphi_{n}(s)\right\|_{W_{n}}^{\tau_{n}}\|s\|_{m_{n}}^{1-\tau_{n}}
$$

and it remains to show that there is $D_{n}>0$ such that $\left\|\varphi_{n}(s)\right\|_{W_{n}} \leq D_{n}\|s\|_{n-1}$. 
This, however, follows from $\left\|\varphi_{n}(s)\right\|_{V_{j} \cap V_{n}}=\left\|\Phi_{n, j} \varphi_{j}(s)\right\|_{V_{j} \cap V_{n}}$ where $\Phi_{n, j}$ is a real analytic function on $Y_{j} \cap Y_{n}$ with values in $G L(p, \mathbb{C})$. By our choice of the $V_{j}$ it is bounded on $V_{j} \cap V_{n}$. Therefore we have

$$
\begin{aligned}
\left\|\varphi_{n}(s)\right\|_{W_{n}} & =\sup _{j=1, \ldots, n-1}\left\|\Phi_{n, j} \varphi_{j}(s)\right\|_{V_{j} \cap V_{n}} \\
& \leq D_{n} \sup _{j=1, \ldots, n-1}\left\|\varphi_{j}(s)\right\|_{V_{j} \cap V_{n}} \\
& \leq D_{n}\|s\|_{n-1},
\end{aligned}
$$

which completes the proof.

We are now ready to prove our first main result.

THEOREM 6. If $F \subset A_{E}(X)$ is a complemented subspace, which is a Fréchet space in the induced topology, then $F$ is finite dimensional.

Proof. We show that $F$ has properties $(\overline{\bar{\Omega}})$ and $(\underline{D N})$. Then we conclude by use of [10], as in [2], that $F$ must be a Banach space hence, being nuclear, is finite dimensional.

The vector bundle $E$ extends to a complex analytic vector bundle on a neighborhood of $X$ in $Y$ which we may write as the intersection of a holomorphically convex neighborhood of $\mathbb{C}^{N}$ with $Y$. Hence we may assume that $E$ is the restriction to $X$ of a complex analytic vector bundle $\hat{E}$ on $Y$. Let $\mathscr{G}$ be the sheaf of sections of $\hat{E}$. Then $A_{E}(X)=G(X)$.

Since $F$ is a quotient of $A_{E}(X)=G(X)$ Theorem 4 implies that $F$ has property $(\overline{\bar{\Omega}})$.

On the other hand we conclude as in the proof of [2, Lemma 3.5] that there is an open neighborhood $Y^{\prime}$ of $X$ in $Y$ such that the imbedding $F \hookrightarrow G(X)$ factorizes through $G\left(Y^{\prime}\right)$. We may assume that $Y^{\prime}=Y \cap \Omega^{\prime}$ where $\Omega^{\prime}$ is a holomorphically convex neighborhood of $\mathbb{R}^{N}$. Since $\mathscr{G}$ is locally free, Lemma 5 implies that $F$ has property $(\underline{D N})$.

Let $\left(E_{0}, \pi_{0}, X\right)$ and $\left(E_{1}, \pi_{1}, X\right)$ be $q_{j}$-dimensional real analytic vector bundles, $j=$ 0,1 . Let $\mathscr{E}_{E_{0}}(X)$ and $\mathscr{D}_{E_{1}}^{\prime}(X)$ denote the $C^{\infty}$ - resp. distributional sections on $X$ and let $L$ denote a differential operator acting from $\mathscr{E}_{E_{0}}(X)$ to $\mathscr{D}_{E_{1}}^{\prime}(X)$. We call it elliptic if $\operatorname{ker} L \subset A_{E_{0}}(X)$.

LEMma 7. If $L$ is elliptic then ker $L$ is a Fréchet space in the topology induced by $A_{E_{0}}(X)$.

Proof. ker $L$ is a Fréchet space if equipped with the topology induced by $\mathscr{E}_{E_{0}}(X)$, and it is a webbed space if equipped with the stronger topology induced by $A_{E_{0}}(X)$, since $A_{E_{0}}(X)$ is webbed and ker $L$ is a closed subspace. By de Wilde's open mapping theorem (see [7, Theorem 24.30]) both topologies coincide hence ker $L$ is a Fréchet space in the topology induced by $A_{E_{0}}(X)$.

In Poly [8, Corollaire 1] it was shown for elliptic $L$, in generalization of a theorem of Grothendieck (see [9]), that ker $L$ is finite dimensional if it is complemented in $\mathscr{E}_{E_{0}}(X)$. The proof was based essentially on the principle of analytic continuation. Our proof based on the use of linear topological invariants gives a sharper result.

THEOREM 8. If $L$ is elliptic and $\operatorname{ker} L$ is complemented in $A_{E_{0}}(X)$ then it is finite dimensional.

Proof. The result is an immediate consequence of Lemma 7 and Theorem 6 . 
Consequently, an infinite dimensional ker $L$ is not complemented in all locally convex spaces containing $A_{E_{0}}(X)$ as continuously imbedded subspace. More precisely:

THEOREM 9. If $H$ is locally convex and $A_{E_{0}}(X) \subset H$ with continuous imbedding, $L$ elliptic and $\operatorname{ker} L$ complemented in $H$, then $\operatorname{ker} L$ is finite dimensional.

Proof. Let $P$ be a continuous projection in $H$ onto ker $L$, then the restriction $P_{0}$ of $P$ to $A_{E_{0}}(X)$, acting from the ultrabornological space $A_{E_{0}}(X)$ to ker $L$ with its Fréchet topology has closed graph, hence is continuous (see [7, Theorem 24.31]). Therefore $P_{0}$ is a projection in $A_{E_{0}}(X)$. From Theorem 8 follows the result.

Let $\mathscr{R}$ denote any regularity class so that, in self explaining terminology, $A_{E_{0}}(X) \subset$ $\mathscr{R}_{E_{0}}(X)$, and let $L$ act from $\mathscr{R}_{E_{0}}(X)$ to any locally convex space $F$. If $L$ is elliptic and ker $L$ is infinite dimensional, then $L$ has no continuous linear right inverse from its range space to $\mathscr{R}_{E_{0}}(X)$, in particular, it is not a "morphisme direct" in the sense of [8].

Finally we extend the principal result of [2] to our much more general situation.

THEOREM 10. If $X$ is non-compact, then $A_{E}(X)$ has no basis.

Proof. By Lemma 2 the nuclear (DF)-space $A_{E}(X)$ is ultrabornological and by Theorem 6 is has no non-trivial complemented Fréchet subspaces. Assume that it has a basis. Then, by [2, Theorem 2.2], it is a (DF)-space. Now, let $s_{0} \in A_{E}(X), s_{0} \neq 0$. Then there is a discrete sequence $z_{1}, z_{2}, \ldots$ with $s_{0}\left(z_{n}\right) \neq 0$ for all $n$. Let $\eta_{n} \in \pi^{-1}\left\{z_{n}\right\}^{*}$ with $\eta_{n}\left(s_{0}\left(z_{n}\right)\right) \neq 0$. Then the map $A(X) \rightarrow \mathbb{C}^{\mathbb{N}}$ defined by $f \mapsto\left(\eta_{n}\left(\left(f s_{0}\right)\left(z_{n}\right)\right)\right)_{n \in \mathbb{N}}$ is surjective. Therefore the map $s \mapsto\left(\eta_{n}\left(s\left(z_{n}\right)\right)\right)_{n \in \mathbb{N}}$ is a continuous linear surjective map from the (DF)-space $G(X)$ to the Fréchet space $\mathbb{C}^{\mathbb{N}}$. This is impossible.

\section{References}

[1] H. Cartan, Variétés analytiques réelles et variétés analytiques complexes, Bull. Soc. Math. France 85 (1957), 77-99.

[2] P. Domański and D. Vogt, The space of real analytic functions has no basis, Studia Math. 142 (2000), 187-200.

[3] H. Grauert, On Levi's problem and the imbedding of real-analytic manifolds, Ann. of Math. 68 (1958), 460-472.

[4] R. C. Gunning and H. Rossi, Analytic Functions of Several Complex Variables, Prentice Hall, Englewood Cliffs, 1965.

[5] M. Klimek, Pluripotential Theory, Clarendon Press, Oxford, 1991.

[6] S. G. Krantz and H. R. Parks, A Primer of Real Analytic Functions, Birkhäuser, BostonBasel-Berlin, 2nd edition, 2002.

[7] R. Meise and D. Vogt, Introduction to Functional Analysis, Clarendon Press, Oxford, 1997.

[8] J.-B. Poly, Sur les opérateurs différentiels et les morphismes directs, C. R. Acad. Sc. Paris 270 (1970), Série A, 647-649.

[9] F. Trèves, Locally Convex Spaces and Linear Partial Differential Equations, Appendix C, Springer, 1967. 
[10] D. Vogt, Frécheträume, zwischen denen jede stetige lineare Abbildung beschränkt ist, J. Reine Angew. Math. 345 (1983), 182-200.

[11] D. Vogt, Topics on projective spectra of (LB)-spaces, in: Advances in the Theory of Fréchet Spaces, T. Terzioğlu (ed.), NATO Adv. Sci. Inst. Ser. C: Math. Phys. Sci. 287 (1989), $11-27$.

[12] J. Wengenroth, Derived Functors in Functional Analysis, Lect. Notes Math. 1810, Springer, Berlin, 2003.

[13] V. P. Zahariuta, Spaces of analytic functions and complex potential theory, in: Linear Topological Spaces and Complex Analysis 1, A. Aytuna (ed.), METU-TÜBİTAK, Ankara 1994, 74-146. 
